\title{
Psychological Aspects of Diabetes
}

\author{
Authors: \\ Naseer Ahmad Bhat, *Krishna Prasad Muliyala, Santosh Kumar \\ Chaturvedi \\ Department of Psychiatry, National Institute of Mental Health and Neurosciences, \\ Bangalore, India \\ *Correspondence to krishnadoc2004@gmail.com \\ Disclosure: $\quad$ The authors have declared no conflicts of interest. \\ Received: $\quad 10.07 .20$ \\ Accepted: $\quad 04.09 .20$ \\ Keywords: $\quad$ Cognitive dysfunction, diabetes, personality, psychological aspects, psychological \\ interventions. \\ Citation: $\quad$ EMJ Diabet. 2020;8[1]:90-98.
}

\section{Abstract}

Diabetes is fundamentally a chronic metabolic disorder, yet it has established psychological connections and consequences. The present article offers an overview of some of the established findings with respect to the psychological aspects of diabetes among adults and adolescents. This narrative review describes the psychological impact of diabetes and the manner in which psychological functioning of the individual affects the development, management, and outcome of diabetes. Diabetes can lead to a great deal of distress, common mental health problems such as anxiety, depression, and sleep disorders, and can increase the risk of suicide. It also affects cognitive functioning across multiple domains such as attention, concentration, memory, executive function, and information processing speed. Diabetes is a burdensome life condition that significantly reduces quality of life. Personality characteristics can have both positive and negative impacts on selfmanagement of diabetes, and some personality profiles, especially the distressed/Type D personality, are indicative of poor prognosis and greater chances of developing medical complications. Psychological interventions such as cognitive behaviour therapy, acceptance and commitment therapy, behavioural activation, and counselling strategies such as educational programmes, problem solving training, and motivational interviewing have proven very effective in coping with diabetes distress, managing comorbid mental health problems, and increasing adherence to self-care and antidiabetic behaviours. Additionally, yogic practices have also shown promising results for selfmanagement of diabetes. Paediatric diabetes especially presents unique psychosocial challenges to patient management and affects academic performance of children and career choices of affected individuals.

\section{INTRODUCTION}

Diabetes, like other chronic, noncommunicable diseases, has a definite psychological impact on the affected individuals and their families. Conversely, the psychological makeup and functioning of the affected individual also affects

several aspects of diabetes morbidity outcomes. The aim of this review article was to provide an update of the major psychological aspects that are affected in patients with diabetes, as well as those that affect the development, management, prognosis, and outcomes of diabetes. This review has used a narrative synthesis approach 
and examined relevant articles from databases such as PsychINFO, PubMed, and Google Scholar. The key search terms used in this review consisted of a standard prefix and a variable suffix term. The prefix across the search terms was 'diabetes mellitus and', and the suffix terms included the following: mental health, depression, anxiety, distress, coping styles, coping, suicide, suicidal ideation, personality characteristics, Type D personality, quality of life (QoL), health-related QoL, psychological interventions, Cognitive Behaviour Therapy (CBT), Acceptance and Commitment Therapy (ACT), behavioural activation, counselling strategies, yoga, and COVID-19 pandemic. The other separate search terms included diabetes in children and adolescents, paediatric diabetes, and issues or challenges in paediatric diabetes. Only relevant articles with a focus on diabetes in relation to the suffix search terms and those published after January 2010 were included in this review.

\section{DIABETES AND COMMON MENTAL HEALTH DISORDERS}

Diabetes not only has physical comorbidities, but also mental health comorbidities such as depression and anxiety, which are common among patients with diabetes. ${ }^{1}$ People with Type 1 and Type 2 diabetes mellitus are three times and two times more likely to develop depression compared to people without diabetes, respectively. ${ }^{2}$ Approximately $30 \%$ of the children and adolescents with Type 1 diabetes mellitus have depressive symptoms. ${ }^{3}$ A cross-national study reported a $10.6 \%$ prevalence of clinical depression and $\mathbf{1 7 . 0 \%}$ prevalence of moderateto-severe depressive symptoms among patients with diabetes. ${ }^{4}$ Additionally, sex, education, diabetes distress, and a history of major depression were found to be risk factors for developing depression. ${ }^{4}$ Patients with diabetes may develop depression because of increased burden of disease management or because of associated biochemical changes accompanying diabetes. Alternatively, patients with depression may have poorer clinical parameters and outcomes of diabetes because of the difficulty in maintaining health behaviours such as increased physical activity, a healthy diet, and medication compliance. Depression may thus be both a consequence as well as a risk factor for diabetes. ${ }^{5}$ Patients with diabetes have been reported to exhibit a 36\% higher risk of developing microvascular complications and 25\% higher risk of macrovascular complications. ${ }^{6}$

Patients with diabetes lead a life that is demanding, constantly challenging, and full of uncertainties. They share a constant concern about maintaining normal levels of blood glucose, medical complications, episodes of hypoglycaemia, hyperglycaemia, and other characteristics of diabetes morbidity. As a result, it is obvious that patients with diabetes may develop symptoms of anxiety or anxiety disorders. A cross-national study revealed that $18.0 \%$ of diabetic patients had at least one type of anxiety disorder and $2.8 \%$ had multiple anxiety disorders; ${ }^{7}$ generalised anxiety disorder was the most common (8.1\%), followed by panic disorder (5.1\%). ${ }^{7}$ Female sex, diabetic complications, longer duration of illness, and glycaemic control were significant risk factors for developing anxiety among patients with diabetes.. ${ }^{7}$

Apart from common mental health issues, patients with diabetes also experience greater sleep-related problems. ${ }^{8}$ For example, 69\% of patients with diabetes had diagnosed breathing-related sleep disorders and $27 \%$ had restless leg syndrome, which are far below the general population prevalence of $2-4 \%$ and $6 \%$, respectively. ${ }^{8}$ Conversely, the risk of developing Type 2 diabetes mellitus was 28\% higher among adults with a sleep duration of $<5-6$ hours per night and $48 \%$ higher among adults with a sleep duration of $>8-9$ hours per night. ${ }^{9}$ The incidence of Type 2 diabetes mellitus was 57\% higher among those who had difficulty in initiating sleep, and this reached $84 \%$ among those who had difficulty in maintaining sleep. ${ }^{9}$ Shorter durations of sleep have been specifically associated with greater incidences of Type 2 diabetes mellitus, poorer glycaemic control, and reduced insulin sensitivity. ${ }^{10}$ However, these findings are not conclusive as the results vary with the use of objective parameters versus self-report.

\section{DIABETES DISTRESS AND COPING}

Diabetes distress refers to a range of negative emotional states that arise from diabetes morbidity and self-care behaviours that patients 
engage in for better management of their diabetes." Diabetes distress is not the same as depression (Table 1), and the two conditions do exist simultaneously and independently. ${ }^{12}$ Diabetes distress is largely an emotional response to the challenges posed by diabetes and may include emotional reactions such as fear, worry, anger, guilt, sadness, frustration, and burn out. ${ }^{2}$ On the other hand, depression involves significant cognitive, affective, social, motivational, and vegetative disturbances in an individual. Both have been reported to have distinct outcomes and associations with clinical parameters related to diabetes self-care. Diabetic patients with comorbid depression are more likely to develop clinical complications compared to non-depressed patients. ${ }^{6}$ Similarly, diabetic distress has been associated with cross-sectional and time-concordant levels of haemoglobin A1C (HbA1c) among adults, whereas no such association has been found between depressive symptoms or clinical depression and HbA1c.13,14 Diabetes distress, rather than depressive symptoms, predict self-reported HbA1c levels among adolescents. ${ }^{15}$
The sources of diabetic distress can be multiple, and one study has factor analytically derived seven major sources of distress in diabetic patients (Table 2): powerlessness, negative social perceptions, physician distress, friend/family distress, hypoglycaemia distress, management distress, and eating distress. ${ }^{16}$ Severity of diabetic distress is directly associated with longer duration of illness, elevated levels of $\mathrm{HbA1c}$, higher BMI, decreased social support, younger age, excessive sleepiness during day time, and lower self-efficacy. ${ }^{17-19}$ Management of diabetes distress is important as unmanaged distress is associated with poor glycaemic control, medication adherence issues, decreased QoL, lower self-efficacy, negative health beliefs, and poor self-care behaviours. ${ }^{13,15,20-21}$

Psychoeducational approaches that address both diabetes and emotion have been reported to be effective for diabetes distress; 22 this is preferably delivered by a generalist. A group format may also be effective, ${ }^{22}$ whereas motivational interviewing, though useful for several long-term conditions, has been reported to be equivocal in the management of diabetes distress. ${ }^{22}$

Table 1: Differentiating between diabetes distress and depression.

\begin{tabular}{|c|c|}
\hline Diabetes distress & Depression \\
\hline $\begin{array}{l}\text { Mainly an affective response to diabetes morbidity and } \\
\text { burden of the disease }\end{array}$ & $\begin{array}{l}\text { A complex response and involves a range of other } \\
\text { reactions dissimilar from the affective response }\end{array}$ \\
\hline $\begin{array}{l}\text { Specific affective reactions may include worry, fear, guilt, } \\
\text { sadness, anger, frustration, and burnout }\end{array}$ & $\begin{array}{l}\text { Response usually includes cognitive, affective, social, } \\
\text { motivational, vegetative, and interpersonal disturbances }\end{array}$ \\
\hline Prevalence is greater & Prevalence is relatively lesser \\
\hline Diabetes and diabetic distress seem to be linearly related & $\begin{array}{l}\text { Diabetes and depression seem to have reciprocal } \\
\text { connections in many cases }\end{array}$ \\
\hline $\begin{array}{l}\text { Not a significant risk factor for developing medical } \\
\text { complications }\end{array}$ & $\begin{array}{l}\text { Is a significant risk factor for developing medical } \\
\text { complications }\end{array}$ \\
\hline $\begin{array}{l}\text { Has been a relatively consistently associated with } \mathrm{HbA1c} \\
\text { levels }\end{array}$ & $\begin{array}{l}\text { Has not been shown to have consistent associations with } \\
\mathrm{HbA1c} \text { levels }\end{array}$ \\
\hline $\begin{array}{l}\text { Interventions may involve psychoeducation, supportive } \\
\text { therapy, counselling, and other simple behaviour } \\
\text { management methods }\end{array}$ & $\begin{array}{l}\text { Interventions may involve use of complex psychological } \\
\text { interventions such as CBT and ACT }\end{array}$ \\
\hline
\end{tabular}

ACT: acceptance and commitment therapy; CBT: cognitive behavioural therapy. 
Table 2: Seven factor analytically derived sources of diabetic distress.

\begin{tabular}{|c|c|}
\hline Source of distress & Description \\
\hline Powerlessness & $\begin{array}{l}\text { A state of helplessness when individuals unsuccessfully try } \\
\text { to control several challenging, and often uncontrollable, } \\
\text { aspects of diabetes }\end{array}$ \\
\hline Negative social perceptions & $\begin{array}{l}\text { Feelings of social mistreatment and discrimination by } \\
\text { people and employers }\end{array}$ \\
\hline Physician distress & $\begin{array}{l}\text { Feelings of mistrust and incompetence about the physician } \\
\text { treating diabetes }\end{array}$ \\
\hline Friend/family distress & $\begin{array}{l}\text { Feeling of being treated as sick and different by family } \\
\text { members and friends. Feeling that family and friends } \\
\text { exaggerate the threat posed by diabetes }\end{array}$ \\
\hline Hypoglycaemia distress & $\begin{array}{l}\text { Fearful feelings of experiencing sudden episodes of } \\
\text { hypoglycaemia such as during driving or sleeping, and fear } \\
\text { of failing to notice signs of hypoglycaemia }\end{array}$ \\
\hline Management distress & $\begin{array}{l}\text { Feeling distressed over not constantly monitoring one's } \\
\text { blood glucose levels and feelings of not being sufficiently } \\
\text { considerate to diabetes care }\end{array}$ \\
\hline Eating distress & $\begin{array}{l}\text { Feeling distressed over unhealthy eating and not } \\
\text { exercising disciplined eating behaviour to support better } \\
\text { management of diabetes }\end{array}$ \\
\hline
\end{tabular}

Fisher et al. ${ }^{16}$ used qualitative interviews and factor analytic procedures to derive seven major sources of diabetes distress among adults with Type 1 diabetes mellitus.

\section{COGNITIVE DYSFUNCTION AND DIABETES}

Diabetes is accompanied by dysfunction in both basic- and higher-order domains of a patient's cognitive functioning. ${ }^{23}$ A systematic review of cognitive functioning in Type 2 diabetes mellitus revealed significantly poorer performance in six domains: motor function, executive function, processing speed, verbal and visual memory, and concentration. ${ }^{24}$ The effect size difference between diabetic and nondiabetic individuals was small-to-moderate for most of these cognitive domains, and smallest for the attention concentration domain. ${ }^{24}$

The cognitive dysfunction in diabetes may manifest in a very subtle form known as diabetes-associated cognitive decrements (DACD). DACD does not cause significant disruption in the daily activities of patients with diabetes and hence is not considered abnormal enough to warrant formal neuropsychological assessment. $^{23}$ The relatively noticeable cognitive impairment in diabetes is subsumed under mild cognitive impairment $(\mathrm{MCl})$, which is regarded as a transitional state between DACD and severe forms of cognitive impairment such as dementia. ${ }^{23}$ A subtype of $\mathrm{MCl}$, amnesic $\mathrm{MCl}$, is concerned with memory-related issues and forgetting, and leads to noticeable disruption in a patient's life, which necessitates formal neuropsychological testing and management. Severe cognitive impairment in diabetes is diagnosed when cognitive dysfunction progresses to the level of formal cognitive disorders such as dementia. It is associated with significant impairment in multiple domains of cognitive functioning and causes a significant disruption to instrumental daily activities.

Cognitive dysfunction has shown associations with clinical characteristics and management of diabetes. Patients using metformin have been shown to have greater risk of cognitive impairment and exhibit decreased cognitive performance compared to those not using 
metformin. ${ }^{25}$ Uncontrolled glycaemic levels also present a significant risk factor for cognitive impairment. Longer duration of illness and early age of onset have been associated with development of dementia or a greater risk of progression from mild to severe cognitive impairment among patients with diabetes. ${ }^{26-27}$ Apart from clinical characteristics, risk and severity of cognitive dysfunction also varies with sociodemographic characteristics such that dysfunction is severe among older patients with diabetes and less severe among patients with higher levels of education. ${ }^{28}$

Cognitive dysfunction in diabetes has multiple potential underlying bases which include uncontrolled glycaemia, decreased functional connectivity of working memory networks, vascular disturbances at micro and macro levels, and disturbances in insulin signalling. ${ }^{29,30}$ Specifically, insulin signalling is believed to influence long-term potentiation in the hippocampus, which has a high density of insulin receptors and is involved in learning and memory. ${ }^{29,30}$ Insulin also affects the levels of neurotransmitters, such as acetylcholine, epinephrine, and norepinephrine, that are implicated in memory functioning. ${ }^{29}$

\section{DIABETES AND QUALITY OF LIFE}

QoL is a broad and multidimensional construct that refers to an individual sense of general wellbeing. Diabetes is a burdensome life condition and patients face significant issues and challenges at the physical, emotional, psychological, social, occupational, and interpersonal levels. Constant monitoring and maintaining of normal blood glucose levels, consistent use of antidiabetic drugs, fear of hyper or hypoglycaemic episodes, fear of developing medical complications, psychiatric comorbidities, restricted food choices, travel constraints, obligation to routine physical exercises, financial costs, mobility issues, and reduced social interactions can be overwhelming. ${ }^{31}$ As a result, individuals living and dealing with diabetes experience a range of negative emotions such as worry, fear, anger, guilt, sadness, helplessness, hopelessness, frustration, and burnout, which all considerably decrease their QoL."
In view of the decreased QoL, the focus of diabetes treatment is not just to control glycaemic levels and prevent medical complications, but also to improve QoL by lessening the overall burden of disease. Besides improvement in therapeutics, additional and more affordable options for medical care, effective psychological and educational interventions for diabetes management, and the presence of support groups have considerably improved the quality of patient's life. ${ }^{32}$ There is a greater need for assessment of QoL in diabetes care and research has led to the development of tools such as the Audit of Diabetes-Dependent QoL (ADDQOL) and Diabetes QoL (DQoL). ${ }^{33}$

\section{DIABETES AND PERSONALITY CHARACTERISTICS}

Management of diabetes, like many other chronic illnesses, is affected by personality characteristics. Certain personality characteristics are known to interfere with self-care behaviours, coping with diabetes distress, and affect the diabetes outcome. The personality profile of individuals with diabetes has been examined using the framework of the five-factor model. ${ }^{34}$ The five factors include openness, conscientiousness, extraversion, agreeableness, and neuroticism. Conscientiousness is strongly associated with better diabetes selfcare, especially glycaemic control. ${ }^{34}$ This is because conscientious individuals are more organised and less impulsive and hence exhibit consistency in self-caring behaviours such as glucose monitoring, physical activity, regular visits to a physician, and effective control over impulsive eating. Patients who exhibit higher levels of neuroticism, an index of emotional instability, tend to worry persistently; are anxious, fearful, and overthink; and have persistently negative moods. It is likely to interfere with self-management, adherence, and may also contribute to increased likelihood of developing psychiatric comorbidities among patients with diabetes. ${ }^{34,35}$

Another thread of research has focussed on distressed/Type D personality, characterised by higher levels of negative affectivity, social inhibition, and constricted expression of negative emotions in social interactions. ${ }^{36} 52 \%$ of patients with diabetes were found to have Type 
D traits that have significant implications for diabetes self-care and clinical outcomes of the disease. ${ }^{37-38}$ Type $D$ patients have been shown to struggle with treatment compliance, especially medication adherence and routine visits to the physician. ${ }^{37-38}$ They exhibit a relatively lacklustre attitude towards maintaining antidiabetic behaviours such as adherence to physical activity recommendations, avoiding consumption of high calorie foods, emotional eating, control over BMI, and cholesterol levels. ${ }^{37}$ These individuals have greater chances of experiencing mental health issues such as depression and anxiety. ${ }^{37}$ Type D personality is also an indicator of poor prognosis and adverse clinical consequences of diabetes. ${ }^{39}$

\section{SUICIDE AND DIABETES}

Patients with diabetes have a significantly higher risk of suicidal ideation, attempted suicide, and completed suicide. ${ }^{40}$ The prevalence for suicidal ideation is $16.2 \%$, much higher than the $9.2 \%$ found in the general population. ${ }^{40}$ Annually, approximately 94,000 completed cases of suicide occur worldwide among patients with diabetes. ${ }^{41}$ The reasons for higher rates of suicidal ideation or suicide can be multiple and may include comorbid depression, which is a significant risk factor for suicide. ${ }^{4,6}$ Additionally, patients with diabetes may be overwhelmed by the extreme burden of the disease: taxing diabetes care, financial costs, poor QoL, deterioration in interpersonal relationships, negative cognitions such as constant worry or hopelessness, and poor prognosis. Further, the risk of suicide is modified by clinical characteristics of diabetes, sociodemographic characteristic of the patients, presence of comorbid mental health issues, and specific personality traits and coping styles. ${ }^{42}$

\section{PSYCHOLOGICAL INTERVENTIONS AND COUNSELLING STRATEGIES IN DIABETES}

Psychosocial issues surrounding diabetes care have brought a greater focus on psychological interventions in the overall diabetes management. Several psychological interventions such as CBT, ACT, and behavioural activation strategies have been used to target different dimensions of diabetes morbidity. They include symptoms of depression, anxiety, interpersonal functioning, glycaemic control, diabetes specific distress, QoL, adherence to medication regimens, and maintenance of important selfcare activities including physical activity, dietary recommendations, and regular self-monitoring of blood glucose levels. A modified form of CBT known as CBT for adherence and depression (CBT-AD) integrates conventional CBT for depression with CBT designed to improve overall treatment adherence. ${ }^{43}$ CBT-AD consists of a single stand-alone session in the beginning to foster adherence to medical regimens and self-care behaviours. The latter part of CBT-AD consists of four modules of 9-11 sessions that focus on adherence and depression. ${ }^{43}$ These sessions include motivational interviewing and typical CBT methods and techniques such as behavioural activation and activity scheduling, monitoring of mood, glucose levels, dietary and physical activities, monitoring of thoughts, maladaptive cognitions and their restructuring, problem solving, and relaxation exercises. ${ }^{43}$ CBT-AD has been effective for control of glycaemic levels, adherence to medication and self-monitoring of blood glucose, reduction in depression severity, and significant improvement in diabetes self-care behaviours. ${ }^{43-44}$

ACT has shown promising results in improving self-care behaviours and glycaemic control. ${ }^{45}$ Contrary to CBT, the ACT does not attempt to confront or change the content of thoughts and feeling of patients with diabetes. Rather, ACT creates an attitude of acceptance towards distressing thoughts, feelings, emotions, and sensations that result from diabetes morbidity. Less complex psychological interventions such as behavioural activation embedded in exercise programmes for patients with diabetes and depression have shown positive results in terms of greater enjoyment of physical activity and cessation of avoidance behaviours. ${ }^{46}$ Other strategies such as teaching problem solving skills, including the use of technology for diabetes problem solving, have been used in the management of diabetes. ${ }^{47}$

Motivational interviewing, as an independent counselling approach, has been employed to elicit and build motivation for undertaking antidiabetic behavioural changes. ${ }^{48}$ It is an effective method for helping patients with diabetes to overcome the resistance, 
ambivalence, and self-efficacy issues that generally hinder their engagement in self-care behaviours to manage diabetes or prevent the development of poorer diabetic outcomes. ${ }^{49}$ Motivational interviewing is a collaborative venture between care provider and patient that is patient-focussed and patient-directed. ${ }^{48-49} \mathrm{It}$ is different from the traditional didactic approach of educating patients about the importance of behaviour change. The counsellor adopts and guides the patients through a process of change that involves four major sequential steps: engaging, focussing, evoking, and planning. ${ }^{49}$ Motivational interviewing has demonstrated effectiveness in bringing about successful dietary changes, weight loss, glycaemic control, and improvements in BMI among patients with diabetes. ${ }^{50}$

Complementary therapeutic practices such as yoga are also effective for Type 2 diabetes mellitus care and management. Yogic practices improve not only the primary diabetic symptoms but also have beneficial effects on multiple other functions that are adversely affected by diabetes. Yoga has been shown to have a significant improvement on glycaemia control, $\mathrm{HbA1c}$ and fasting blood glucose levels, postprandial blood glucose, lipid levels, and body composition. ${ }^{51-52}$ Yoga has also been found to be associated with reductions in BMI, anxiety, depression, and oxidative stress, and enhanced cognitive functioning, improved blood pressure, QoL, and general wellbeing. ${ }^{53-55}$

\section{PSYCHOSOCIAL ISSUES IN PAEDIATRIC DIABETES}

The management of diabetes in children and adolescents presents unique challenges; specifically, its management is complicated by developmental changes taking place, their need for autonomy and psychosocial immaturity, peer pressure, and family dynamics. ${ }^{56}$ Nonadherence to medical regimens such as insulin therapy, as well as other self-care behaviours such as not attending physical activity classes, refusing dietary changes, and maladaptive behaviours, may be observed. ${ }^{56}$ Risk-taking behaviours such as substance abuse and eating disorders can complicate the management process, or may worsen diabetes outcomes. Children in families with dysfunctional social interaction patterns have been shown to exhibit poorer control of certain diabetes parameters. Type 1 diabetes mellitus not only affects children and adolescents directly, but it also has a significantly negative impact on the family. Paediatric diabetes leads to increased stress among parents, conflicts over mismanagement of children's diabetes, fear of adverse diabetes-related complications such as hypoglycaemia, hyperglycaemia, ketoacidosis, and hospitalisations. ${ }^{56,57}$

Paediatric diabetes is also associated with experiencing diabetic distress, anxiety, depression, eating disorders, externalising problems, cognitive dysfunction, and overall reduced QoL. It also affects the performance of children and adolescents in academic as well as nonacademic domains such as sports and exercise, and has adverse implications for their employment and career prospects. ${ }^{58}$ Children with early-onset diabetes and longer duration of illness are particularly at a higher risk for these negative outcomes. ${ }^{58}$

\section{DIABETES, COVID-19, AND MENTAL HEALTH}

Patients with diabetes have been uniquely affected by the current coronavirus disease (COVID-19) pandemic. Complete lockdowns, restrictions on travel and movement, and general anxiety about the pandemic directly and indirectly have all affected clinical aspects of diabetes and self-care. Access to medical services such as physician consultations, antidiabetic drugs, testing and monitoring services, and self-care behaviours such as outdoor physical activities, were all differentially hampered.59 As a result, the COVID-19 pandemic affected glycaemic control of diabetic patients, which was associated with poorer clinical outcomes such as the need for intensive care and even death. ${ }^{60}$ The anxiety caused by lack or irregularity of medical services, fear of being vulnerable to poorer COVID-19 outcomes, and greater mortality rates may have added to pre-existing diabetes distress and further exacerbated the mental health issues of patients with diabetes. 


\section{SUMMARY AND CONCLUSION}

have been affected by the current COVID-19 pandemic, which may lead to poor outcomes. In Patients with diabetes have manifold risk of developing common mental health conditions such as anxiety and depression. A significantly greater proportion of patients experience diabetes distress in response to disease burden and their perceived threats of the disease. Patients with diabetes also experience cognitive dysfunction ranging from very subtle to very severe levels. Additionally, diabetes significantly reduces the QoL of patients. Both patients with diabetes and with mental health disorders

addition to medical management, psychological interventions and counselling strategies have been developed or adapted to deal with diabetes distress, mental health issues, poor QoL, difficulties in self-management of diabetes, and adherence to medical regimens and antidiabetic behaviours. Core psychological therapies such as CBT, ACT, counselling strategies, motivational interviewing, and yogic practices have proven effective for glycaemic control, improved adherence to medication, self-care behaviours, reducing depression severity.

\section{References}

1. Balhara YP. Diabetes and psychiatric disorders. Indian J Endocrinol Metab. 2011;15(4):274

2. Roy T, Lloyd CE. Epidemiology of depression and diabetes: a systematic review. J Affect Disord. 2012;142:S8 21

3. Buchberger B et al. Symptoms of depression and anxiety in youth with Type 1 diabetes: a systematic review and meta-analysis.

Psychoneuroendocrinol. 2016;70:7084.

4. Lloyd CE et al. Prevalence and correlates of depressive disorders in people with Type 2 diabetes: results from the International Prevalence and Treatment of Diabetes and Depression (INTERPRET-DD) study, a collaborative study carried out in 14 countries. Diabet Med. 2018;35(6):760-9

5. Renn BN et al. The bidirectional relationship of depression and diabetes: a systematic review. Clin Psychol Rev. 2011;31(8):1239-46.

6. Lin $\mathrm{EH}$ et al. Depression and advanced complications of diabetes: a prospective cohort study. Diabetes Care. 2010;33(2):264-9

7. Chaturvedi SK et al. More anxious than depressed: prevalence and correlates in a 15-nation study of anxiety disorders in people with Type 2 diabetes mellitus. General Psychiatry. 2019;32(4).

8. Khalil $\mathrm{M}$ et al. The association between sleep and diabetes outcomes-a systematic review. Diabetes Res Clin Pract. 2020;161:108035.

9. Cappuccio FP et al. Quantity and quality of sleep and incidence of Type 2 diabetes: a systematic review and meta-analysis. Diabetes Care. 2010;33(2):414-20.
10. Ogilvie RP, Patel SR. The epidemiology of sleep and diabetes. Curr Diab Rep. 2018;18(10):82.

11. Hagger $\vee$ et al. Diabetes distress among adolescents with Type 1 diabetes: a systematic review. Curr Diab Rep. 2016;16(1):9.

12. Fisher $L$ et al. Impact of baseline patient characteristics on interventions to reduce diabetes distress: the role of personal conscientiousness and diabetes selfefficacy. Diabet Med. 2014;31(6):739 46.

13. Fisher $L$ et al. The relationship between diabetes distress and clinical depression with glycemic control among patients with Type 2 diabetes. Diabetes Care. 2010:33(5):1034-6.

14. Strandberg RB et al. Relationships of diabetes-specific emotional distress, depression, anxiety, and overall wellbeing with $\mathrm{HbA} 1 \mathrm{c}$ in adult persons with Type 1 diabetes. J Psychosom Res. 2014;77(3):174-9.

15. Hagger Vet al. Diabetes distress is more strongly associated with $\mathrm{HbA1c}$ than depressive symptoms in adolescents with Type 1 diabetes: results from Diabetes MILES Youth-Australia. Pediatric Diabetes. 2018;19(4):840-7.

16. Fisher $L$ et al. Understanding the sources of diabetes distress in adults with Type 1 diabetes. J Diabetes Complications. 2015;29(4):572-7.

17. Tan ML et al. Factors associated with diabetes-related distress over time among patients with T2DIABETES in a tertiary hospital in Singapore. BMC Endocr Disord. 2017;17(1):36.

18. Wardian J, Sun F. Factors associated with diabetes-related distress: implications for diabetes selfmanagement. Soc Work Health Care. 2014;53(4):364-81.
19. Aljuaid MO et al. Diabetes-related distress assessment among Type 2 diabetes patients. J Diabetes Res. 2018;7328128:1-10.

20. Gonzalez JS et al. Distress and Type 2 diabetes-treatment adherence: mediating role for perceived control. Health Psychol. 2015;34(5):505.

21. Fisher $L$ et al. Diabetes distress but not clinical depression or depressive symptoms is associated with glycemic control in both crosssectional and longitudinal analyses. Diabetes Care. 2010;33(1):23-8.

22. Sturt $J$ et al. Effective interventions for reducing diabetes distress: systematic review and meta-analysis. Int Diabetes Nurs. 2015;12:40-55.

23. Biessels GJ, Despa F. Cognitive decline and dementia in diabetes mellitus: mechanisms and clinical implications. Nature Rev Endocrinol. 2018;14(10):591-604

24. Palta $P$ et al. Magnitude of cognitive dysfunction in adults with Type 2 diabetes: a metaanalysis of six cognitive domains and the most frequently reported neuropsychological tests within domains. J Int Neuropsychol Soc. 2014;20(3):278-91.

25. Moore EM et al Increased risk of cognitive impairment in patients with diabetes is associated with metformin. Diabetes Care. 2013;36(10):2981-7

26. Yuan $X Y$, Wang $X G$. Mild cognitive impairment in Type 2 diabetes mellitus and related risk factors: a review. Rev Neurosc. 2017;28(7):71523

27. Pal $\mathrm{K}$ et al. Mild cognitive impairment and progression to dementia in people with diabetes, prediabetes and metabolic syndrome: a systematic review and meta-analysis. Soc Psychiatry Psychiatr Epidemiol. 


\section{8:53(11):1149-60}

28. Sun $L$ et al. Risk factors for cognitive impairment in patients with Type 2 diabetes. J Diabetes Res. 2020:4591938:1-10.

29. Zilliox LA, Chadrasekaran K et al. Diabetes and cognitive impairment. Curr Diab Rep. 2016;16(9):87.

30. Zhou $Y$ et al. Clinical characteristics for the relationship between Type2 diabetes mellitus and cognitive impairment: a cross-sectional study. Aging Dis. 2015;6(4):236.

31. Jing $X$ et al. Related factors of quality of life of Type 2 diabetes patients: a systematic review and metaanalysis. Health Qual Life Outcomes. 2018;16(1):189.

32. Trikkalinou A et al. Type 2 diabetes and quality of life. World J Diabetes. 2017;8(4):120-9

33. Nair R, Kachan P. Outcome tools for diabetes-specific quality of life: study performed in a private family practice clinic. Canadian Fam Physic. 2017;63(6):e310-5.

34. Esmaeilinasab $M$ et al. Type II diabetes and personality; a study to explore other psychosomatic aspects of diabetes. J Diabetes \& Metab Disord. 2016;15(1):54.

35. Novak JR et al. Does personality matter in diabetes adherence? Exploring the pathways between neuroticism and patient adherence in couples with Type 2 diabetes Applied Psychol Health Well-Being. 2017;9(2):207-27.

36. van Dooren FE et al. Psychological and personality factors in Type 2 diabetes mellitus, presenting the rationale and exploratory results from The Maastricht Study, a populationbased cohort study. BMC Psychiatr. 2016;16:17.

37. Conti $\mathrm{C}$ et al. The clinical link between Type D personality and diabetes. Front Psychiatry. 2016;7:113.

38. Li $X$ et al. Type $D$ personality predicts poor medication adherence in Chinese patients with Type 2 diabetes mellitus: a six-month follow-up study. Plos One. 2016;11(2)

39. Shao Yet al. Type D personality as a predictor of self-efficacy and social support in patients with Type 2 diabetes mellitus. Neuropsychiatr Dis Treat. 2017;13:855

40. Elamoshy $\mathrm{R}$ et al. Risk of depression and suicidality among diabetic patients: a systematic review and meta-analysis. J Clin Med. 2018;7(11):445.

41. Wang B et al. Management of endocrine disease: suicide risk in patients with diabetes: a systematic review and meta-analysis. Eur J Endocrinol. 2017;177(4):R169-81.

42. Sarkar S, Balhara YP. Diabetes mellitus and suicide. Indian Endocrinol Metab. 2014;18(4):468.

43. Gonzalez JS et al. Cognitivebehavioral therapy for adherence and depression (CBT-AD) in Type 2 diabetes. J Cogn Psychother. 2010;24(4):329-43.

44. Safren SA et al. A randomized controlled trial of cognitive behavioral therapy for adherence and depression (CBT-AD) in patients with uncontrolled Type 2 diabetes. Diabetes Care. 2014;37(3):625-33.

45. Shayeghian $Z$ et al. A randomized controlled trial of acceptance and commitment therapy for Type 2 diabetes management: the moderating role of coping styles. Plos One. 2016;11(12):e0166599.

46. Schneider $\mathrm{K}$ et al. Feasibility of pairing behavioral activation with exercise for women with Type 2 diabetes and depression: the get it study pilot randomized controlled trial. Behav Ther. 2016;47(2):198-212

47. Chew BH et al. Psychological interventions for diabetes-related distress in adults with Type 2 diabetes mellitus. Cochrane Database Syst Rev. 2017; DOI: 10.1002/14651858. CD011469.pub2.

48. Salimi $\mathrm{C}$ et al. A review on effectiveness of motivational interviewing in the management of diabetes mellitus. J Psychol Clin Psychiatry. 2016;5(4):1-6.

49. Steinberg M. Clinical perspectives on motivational interviewing in diabetes care. Diabetes Spectrum 2011:24(3):179-81.
50. Ekong G, Kavookjian J. Motivational interviewing and outcomes in adults with Type 2 diabetes: a systematic review. Patient Educ Couns. 2016;99(6):944-52

51. Innes KE, Selfe TK. Yoga for adults with Type 2 diabetes: a systematic review of controlled trials. J Diabetes Res. 2016; DOI: 10.1155/2016/6979370.

52. Thind $\mathrm{H}$ et al. The effects of yoga among adults with Type 2 diabetes: a systematic review and meta-analysis. Prevent Med. 2017;105:116-26.

53. Hegde SV et al. Effect of 3-month yoga on oxidative stress in Type 2 diabetes with or without complications: a controlled clinical trial. Diabetes Care. 2011;34(10):220810.

54. Satish L, Lakshmi VS. Impact of individualized yoga therapy on perceived quality of life performance on cognitive tasks and depression among Type II diabetic patients. Int J Yoga. 2016;9(2):130.

55. Yadav RK et al. A short-term, comprehensive, yoga-based lifestyle intervention is efficacious in reducing anxiety, improving subjective wellbeing and personality. Int J Yoga. 2012;5(2):134.

56. Deeb A et al. Common issues seen in paediatric diabetes clinics, psychological formulations, and related approaches to management. J Diabetes Res. 2018; DOI: 10.1155/2018/1684175.

57. Driscoll KA et al. Fear of hypoglycemia in children and adolescents and their parents with Type 1 diabetes. Curr Diab Rep. 2016;16(8):77

58. Oakley NJ et al. Type 1 diabetes mellitus and educational attainment in childhood: a systematic review. BMJ Open. 2020;10:e033215.

59. Barone MTU et al. The impact of COVID-19 on people with diabetes in Brazil. Diabetes Res Clin Pract. 2020;166:108304.

60. Apicella M et al. COVID-19 in people with diabetes: understanding the reasons for worse outcomes. Lancet Diabetes Endocrinol. 2020:8:782-92. 